\title{
Adaptive adjustment of the number of subsets during iterative image reconstruction
}

\author{
Kris Thielemans, Senior Member, IEEE, Simon Arridge
}

\begin{abstract}
A common strategy to speed-up image reconstruction in tomography is to use subsets, i.e. only part of the data is used to compute the update, as for instance in the OSEM algorithm. However, most subset algorithms do not convergence or have a limit cycle. Different strategies to solve this problem exist, for instance using relaxation. The conceptually easiest mechanism is to reduce the number of subsets gradually during iterations. However, the optimal point to reduce the number of subsets is usually depends on many factors such as initialisation, the object itself, amount of noise etc.

In this paper, we propose a simple scheme to automatically compute if the number of subsets is too large (or too small) and adjust the size of the data to consider in the next update automatically. The scheme is based on idea of computing two image updates corresponding to different parts of the data. A comparison of these updates then allows to see if the updates were sufficiently consistent or not. We illustrate this idea using 2 different subset algorithms: OSEM and OSSPS.
\end{abstract}

\section{INTRODUCTION}

Subset algorithms speed-up the calculations dramatically and are therefore very popular. However, the benefits of using a large number of subsets are usually only present for the initial updates. After a larger number of iterations, the update computed from a subset of the data is not necessarily "optimal", i.e. does not "point" towards the correct solution. It is well-known that OSEM has a limit cycle, and many "simple" subset algorithms have similar behaviour. Relaxation, i.e. gradually decreasing step-size, can solve this problem [Ahn2003] and can lead to convergent algorithms such as RAMLA and relaxed OSSPS. However, the amount of relaxation to apply is not obvious, and this can lead to slow convergence. Many authors therefore prefer to reduce the number of subsets gradually during iterations. However, the optimal point to reduce the number of subsets is usually depends on many factors such as initialisation, the object itself, amount of noise etc.

We propose a simple scheme that compares the update directions for 2 different subsets. If these are too inconsistent, the proposed algorithm reduces the number of subsets automatically, avoiding future updates to be in different directions. We illustrate this idea using 2 different subset algorithms: OSEM and OSSPS.

\section{METHOD}

A. Notations:

$y \quad$ : subdivided in $N$ subsets $y_{S}$

$x^{c} \quad$ : current image estimate

$P, P_{S} \quad$ : forward projection matrix, matrix for the subset $s$

$S=P^{\prime} .1, S_{S}=P_{s}^{\prime} .1$ : sensitivity image (back-projection of data all set to 1$)$, and subset sensitivity

$x_{s}^{\text {new }}=R\left(x^{c} ; y_{s}\right)$ : image update for a subset of the data $m_{s s^{\prime}}=M\left(x_{s}^{\text {new }}, x_{s^{\prime}}^{\text {new }} ; x^{c}\right)$ : metric comparing two image updates for different subsets

$x^{\text {new }}=C\left(x_{s}^{\text {new }}, x_{s^{\prime}}^{\text {new }}\right) \quad:$ combination of 2 image estimates

to obtain a new estimate

\section{B. Algorithm for image update and subset size:}

A refinement to the basic idea is to avoid wasting computation time by combining the updates from both subsets into one update, as opposed to keeping only one update.

0 . Obtain current image estimate $x^{c}$

1. Select 2 subsets that are "orthogonal" in some sense (e.g. views from very different angles)

2. Compute 2 updates and compare them using the metric $M$

3. If $m_{s s}$ is lower than a threshold $t_{\text {min }}$, reduce number of subsets. Otherwise
a. Compute

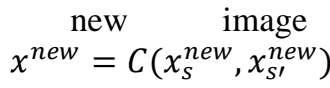
update

b. if $m_{s s}$ is higher than a threshold $t_{\text {max }}$, increase number of subsets.

4. Go to step 1

The metric comparing the updates used here is the cosine between the (additive) updates.

$$
M\left(x_{s}^{\text {new }}, x_{s^{\prime}}^{\text {new }} ; x^{c}\right)=\frac{\left(x_{s}^{n e w}-x^{c}\right) \cdot\left(x_{s^{\prime}}^{n e w}-x^{c}\right)}{\left|x_{s}^{n e w}-x^{c}\right|\left|x_{s^{\prime}}^{n e w}-x^{c}\right|}
$$

To combine the 2 updates for OSEM, a logical weighted averaging uses the subset sensitivities:

$$
C\left(x_{s}, x_{s^{\prime}}\right)=\frac{S_{s} x_{s}^{\text {new }}+S_{s^{\prime}} x_{s^{\prime}}^{\text {new }}}{S_{s}+S_{s^{\prime}}}
$$

This update formula means that the combined update of the 2 candidate updates boils down to the regular OSEM update for the larger subset consisting of data in both subsets $s$ and $s$ '. A similar weighted averaging can be used for OSSPS or any preconditioned gradient descent algorithm.
Manuscript received December 7, 2015. This work was supported by the National Institute for Health Research, Biomedical Research Centre funding (University College London Hospitals).
K. Thielemans is with the Institute for Nuclear Medicine, University College London, UCLH, 235 Euston Road (T-5), London, NW1 2BU, UK (email: k.thielemans@ucl.ac.uk) S. Arridge is with the Centre for Medical Image Computing, University College London, Gower Street, London, WC1E 6BT, UK 


\section{NOTES}

The above formulation recomputes the number of subsets after every image update. So, in a sense, the number of subsets is not relevant, but only the "size" of the subset, i.e. how much data each subset contains. This has the advantage that it is no longer required that the number of subsets divides the number of views (or angles).

\section{RESULTS}

Our results are based on simulated data using the SheppLogan phantom in 2D (120 views). The simulation consisted of line-integral computation with PSF and a small background to represent scatter/randoms. Poisson noise was added after the simulation.

In all reconstructions, we set the threshold $t_{\text {min }}=.5$ and $t_{\max }=.9$. If $t_{\min }$ is reached, we increase subset-size with a factor 1.25 , if $t_{\max }$ is reached, we halved the subset-size. These settings will need further testing.

We compare OSEM with 30 subsets (4 angles per update), EM (120 angles per update) and the proposed OSEM version (OSEMauto) starting with 2 angles in each update-pair. Fig. 1 shows the behaviour of the cosine-metric and the number of subsets used at every update. Fig. 2 shows the MSE and log-likelihood.
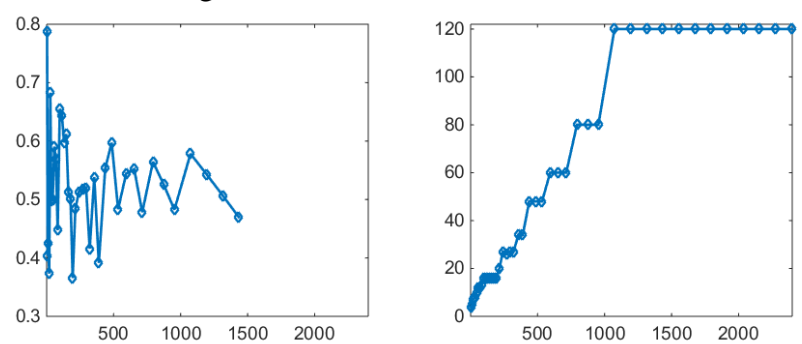

Fig. 1 OSEMauto experiment. Left plot shows the cosine of the angle between the 2 subset updates at each step in the algorithm. Right plot shows the selected number of views per update used. Horizontal axis in both plots is the cumulative number of views used (proportional to computation time).
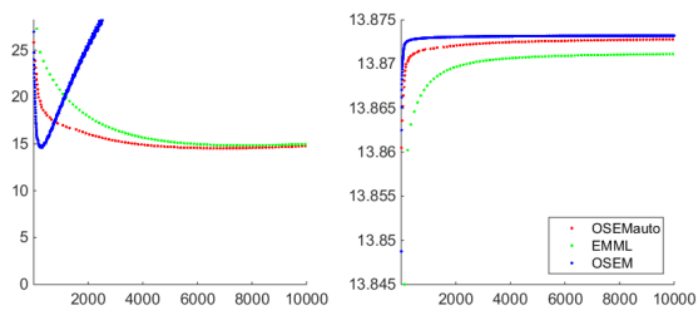

Fig. 2. Left plot shows the evolution over iterations of the Mean Square Error (MSE) between the reconstructed image with OSEM and the true image. Right shows the log-likelihood. The horizontal axis in both plots is the cumulative number of views used.

Similar plots are shown for OSSPS. At each update, we used the original update of Erdoğan et al [1] using only the subset of the data (i.e. not the precomputed denominator approach [2]). A log-cosh prior was used as a penalty.

\section{DISCUSSION}

The proposed algorithm does automatically change subset size. For Maximum Likelihood reconstruction, it is not clear of this is beneficial or not. In practice, OSEM is used with early stopping, so an evaluation in terms of the obtained log-likelihood is not useful. Fig. 2 does show that OSEMauto can obtain a lower MSE than OSEM but with the current settings, it needs far more computations.
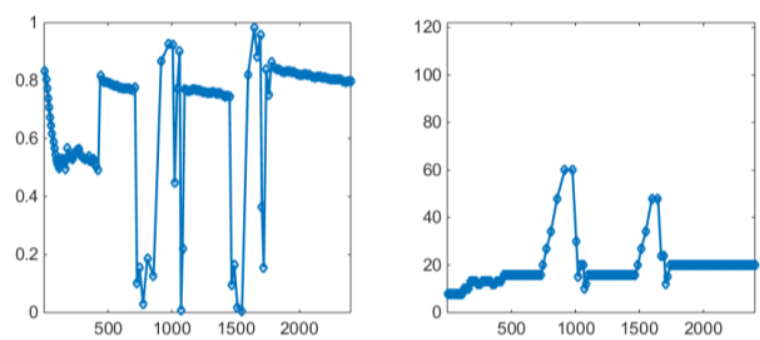

Fig. 3 OSSPSauto experiment. Left plot shows the cosine of the angle between the 2 subset updates at each step in the algorithm. Right plot shows the selected number of views used per update. Horizontal axis in both plots is the cumulative number of views used.
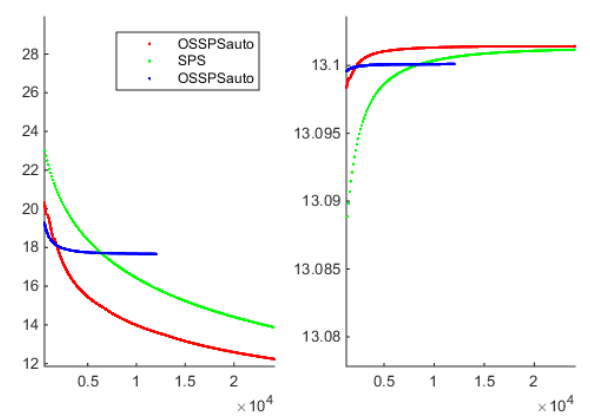

Fig. 4. Left plot shows the evolution over iterations of the Mean Square Error (MSE) between the reconstructed image with OSEM and the true image. Right shows the penalised log-likelihood. The horizontal axis in both plots is the cumulative number of views used.

For OSSPS, the algorithms should ideally maximise the penalised log-likelihood. Although Fig. 3 shows unexpected variations in the subset-size, Fig. 4 shows that OSSPSauto can do this at much lower computational cost than SPS, while OSSPS cannot achieve the same maximum (nor aslow MSE). Note that according to the current criteria, the number of views per update was still small compared to the total number of views even after a large number of updates, indicating that a speed-up is still possible even at large iteration number. It is likely that this behaviour will depend on the penalty. This will be investigated in the future.

\section{CONCLUSION}

The proposed algorithm chooses a subset size that fits the data. Initial results indicate that this is useful for accelerating penalised image reconstruction.

\section{REFERENCES}

[1] H. Erdoğan and J. A. Fessler, Ordered subsets algorithms for transmission tomography, Phys. in Med. Biol., vol. 44, pp. 2835-2851+, 1999

[2] S. Ahn and J. A. Fessler, Globally convergent image reconstruction for emission tomography using relaxed 
ordered subsets algorithms, IEEE Trans. Med. Imag., vol. 22, no. 5, pp. 613-626, May 2003. 